\title{
Influence of Chemical Structure of Some Flavonols on Their Electrochemical Behaviour
}

\author{
Marijan Šeruga*, Ivana Tomac \\ Department of Applied Chemistry and Ecology, Faculty of Food Technology, University of Osijek, \\ Franje Kuhača 20, HR-31000 Osijek, Croatia \\ *E-mail: marijanseruga@gmail.com
}

doi: $10.20964 / 2017.08 .79$

Received: 21 April 2017 / Accepted: 15 June 2017 / Published: 12 July 2017

\begin{abstract}
The electrochemical behaviour of three structurally related flavonols, quercetin, morin, and rutin was studied by cyclic, differential pulse, and square-wave voltammetry methods. The study reveals that their electrochemical behaviour strongly depends on their chemical structure and electronic properties, particularly on the presence of electron-donating-OH groups, i.e. their numbers and position on rings $\mathrm{A}, \mathrm{B}$, and $\mathrm{C}$ in the structure of these flavonols. The important factors of the electrochemical oxidation behaviour of flavonols are as follows. (i) The presence of two electron-donating -OH groups on the $\mathrm{B}$ ring in the ortho-position. (ii). The 2,3-double bond in conjugation with a 4-oxo group on the $\mathrm{C}$ ring. (iii) The electron donating 3-OH group on the $\mathrm{C}$ ring, and (iv) the electron-donating 5-OH and 7-OH groups on A ring. Quercetin satisfies all of the requirements mentioned above and therefore has the best electron-donating properties of all investigated flavonols. The first oxidation peak of quercetin (peak A1) corresponds to the reversible oxidation of $3^{\prime}, 4^{\prime}-\mathrm{OH}$ groups (catechol moiety) at the B ring to the ortho-quinone structure by two-electron-two-proton $\left(2 \mathrm{e}^{-}-2 \mathrm{H}^{+}\right)$process. This electrochemically active and unstable ortho-quinone species then undergoes chemical rearrangements or addition reactions, indicates an electrochemical-chemical (EC) reaction mechanism. At higher anodic potential the $-\mathrm{OH}$ group at position 3 of ring $\mathrm{C}$ was oxidized (peak A2), by the reversible one-electron-oneproton reaction. In this study, it was for the first time observed that the second reduction peak of quercetin (peak $\mathrm{C} 2$ ) corresponds to the 3-OH group on ring $\mathrm{C}$. The hydroxyl groups at position 5 and 7 at ring $\mathrm{A}$ have significantly smaller electron-donating effect than - $\mathrm{OH}$ groups at ring $\mathrm{B}$, and therefore were oxidized at higher anodic potentials (peak A3). This oxidation is an irreversible process. Morin with meta-2',4'-dihydroxyl groups (resorcinol moiety) shows the higher value of oxidation potential of peak A1 than quercetin, indicating that oxidation of 2 ', 4 '-OH groups on ring $\mathrm{B}$ of morin to quinone structure is more difficult in comparison to that of quercetin. This fact clearly shows the importance of the presence of two hydroxyl groups in the ortho-diphenolic arrangement on the ring B of flavonols. The first oxidation process of morin is an one-electron-one-proton reversible reaction, which proceeds in an EC mechanism. The oxidation peak of morin A3 should be associated with the oxidation of 5,7dihydroxyl moiety of ring $\mathrm{A}$, which oxidation occurs at very high oxidation potentials. This oxidation is an irreversible process because no reduction peak was observed. The absence of oxidation peak A2
\end{abstract}


could be explained possibly by the formation of hydrogen bond between the 3-OH group and the oxygen at position $\mathrm{C}-4$ on the ring $\mathrm{C}$. Rutin (quercetin-3-O - rutinose) shows the highest oxidation potential of peak A1 of all investigated flavonols. Such behaviour reflects the influence of glycosylation with rutinose on position 3 of the $\mathrm{C}$ ring. Therefore, an oxidation process was blocked, what significantly decreases the strength of delocalization of electrons from B ring, in comparison to that of quercetin. In concordance with this fact, the oxidation of $\mathrm{B}$ ring of rutin was observed at significantly higher potentials. This result shows how important is the role of 3-OH group in the $\mathrm{C}$ ring on the electrochemical properties of flavonols. The first redox couple of rutin (peaks A1 and C1) corresponds (as in the case of quercetin) to the oxidation of 3',4'-dihydroxy groups on the B ring of rutin and the reduction of the 3',4'-quinone, respectively. This reaction is a reversible process proceeds through EC reaction mechanism. The oxidation peak of rutin A3 should be associated with oxidation of 5,7-dihydroxyl moiety of ring A. This oxidation is an irreversible process because no reduction peak was observed. The absence of oxidation peak A2 could be explained by the presence of rutinoside group without electrochemical activity, at position 3 of $\mathrm{C}$ ring of rutin. The results of present study give some new information about the electrochemical oxidation/reduction processes of investigated flavonols. (i) For the first time was observed the presence of cathodic reduction peak of quercetin (peak $\mathrm{C} 2$ ) which corresponds to $3-\mathrm{OH}$ group at $\mathrm{C}$ ring. (ii) The reversibility of the first oxidation process of morin (peaks A1 and C1) was clearly demonstrated. (iii) It was clearly shown how important is the role of $-\mathrm{OH}$ groups (i.e. their number and their position on rings $\mathrm{A}, \mathrm{B}$, and $\mathrm{C}$ ) on the electrochemical behaviour of investigated flavonols.

Keywords: Electrochemical behaviour, Quercetin, Morin, Rutin, Chemical structure

\section{$\underline{\text { FULL TEXT }}$}

(C) 2017 The Authors. Published by ESG (www.electrochemsci.org). This article is an open access article distributed under the terms and conditions of the Creative Commons Attribution license (http://creativecommons.org/licenses/by/4.0/). 